\title{
Tangence
}

\section{Lecture de l'espace subjectif dans L'invention de la solitude de Paul Auster}

\section{Frances Fortier}

Numéro 43, mars 1994

Babel et après : Paul Auster

URI : https://id.erudit.org/iderudit/025803ar

DOI : https://doi.org/10.7202/025803ar

Aller au sommaire du numéro

Éditeur(s)

Tangence

ISSN

0226-9554 (imprimé)

1710-0305 (numérique)

Découvrir la revue

Citer cet article

Fortier, F. (1994). Lecture de l'espace subjectif dans L'invention de la solitude de

Paul Auster. Tangence, (43), 80-97. https://doi.org/10.7202/025803ar d'utilisation que vous pouvez consulter en ligne.

https://apropos.erudit.org/fr/usagers/politique-dutilisation/ 


\title{
Lecture de l'espace subjectif dans L'Invention de la solitude de Paul Auster
}

\section{Frances Fortier}

\begin{abstract}
Nul n'est moins cynique qu'un magicien. Il sait, et chacun sait, que tout ce qu'il fait est illusion. L'astuce n'est pas vraiment de tromper les gens, mais de les enchanter à un degré tel qu'ils souhaitent être trompés: de sorte que, pendant quelques minutes, la relation. de cause à effet est dénouée, les lois de la nature contredites. (p. 186) ${ }^{1}$
\end{abstract}

De par son titre, L'invention de la solitude instaure d'emblée un pacte de lecture équivoque qui va solliciter à la fois l'efficace de l'illusion référentielle et la mise en scène des procédés mêmes de l'illusion. Certes, il s'agit en l'occurrence d'une histoire archétypale de solitude où le protagoniste, enfermé dans sa chambre, mène une quête identitaire déclenchée par la mort du père. Très tôt, cependant, le récit de soi dévoile ses ficelles et la construction textuelle, l'invention, se désigne par l'exploitation systématique, jusqu'à saturation, des mécanismes autoreprésentatifs. L'écriture austérienne ne se dissimule pas et l'histoire du deuil devient formellement l'écriture de cette histoire. Si ce n'était de l'ampleur des moyens convoqués, l'entreprise, et sa forme, confinerait au cliché. De fait, l'astuce réside précisément dans le tour de plus pascalien, et la mise en récit, loin d'aboutir à la résolution de la recherche d'identité, va accentuer la déréalisation en maintenant l'indétermination du sens et le décentrement du sujet.

D'où l'intérêt d'examiner, par une lecture minutieuse de l'espace subjectif, les pôles tensifs de cette écriture tentée à la fois par la nostalgie du sens et la conscience de son impossibilité.

1 Paul Auster, L'invention de la solitude, Arles, Babel, Coédition Actes Sud, Labor, L'Aire, 1992. Les chiffres entre parenthèses renvoient à la pagination de cette édition. 
Cette lecture entend s'attarder sur trois facettes majeures du sujet, en tant qu'elles sont représentées dans le texte, à savoir le sujet énonciateur, scripteur et lecteur. Espace complexe s'il en est, où la pronominalisation accentue la fragmentation énonciative, où la co-énonciation atomise systématiquement le déroulement diégétique et où l'activité lectorale, constamment commentée, spécifie les registres subjectifs du regard. À l'inventaire des stratégies déictiques succédera donc une investigation du dialogisme textuel, suivie d'une interrogation de la focalisation dans son lien avec la réalité.

À la plate évidence du réel, L'invention de la solitude substitue la sédimentation de l'acte d'écrire et construit patiemment, pour le déconstruire d'un même geste, un espace de représentation délibérément et expressément trafiqué. L'enchantement est à ce prix.

\section{II décide de s'appeler $A$.}

"L'invention de la solitude. Histoires de vie et de mort. L'histoire commence par la fin. Parle ou meurs. Et aussi longtemps que tu parleras, tu ne mourras pas. L'histoire commence par la mort" (p. 232). Cette phrase emblématique de la structure diégétique insiste en outre sur le fait que l'enjeu du texte est sa propre énonciation. Par ailleurs, l'injonction qui assigne au $t u$ la prise de parole signale la fragmentation du sujet, à l'origine même du déroulement narratif. De fait, le moment inaugural de l'énonciation est irrémédiablement lié à la disparition du père: le père, en mourant, plonge son fils dans un monde de ténèbres où les frontières entre la vie et la mort, entre le réel et le rêve, entre soi et les autres sont abolies. Afin d'accéder au statut de sujet, le fils devra écrire le père, enfanter le père par le travail de l'écriture. Impérativement, pour survivre, le fils devra s'énoncer.

La narrativisation de l'énonciation se voit ainsi motivée par la quête d'identité qui structure le texte. D'emblée, la piste est donnée. La première partie de l'ouvrage, intitulée "Portrait d'un homme invisible", met en scène un narrateur-écrivain je qui entend écrire l'histoire du père qui vient de mourir. Dans la seconde partie, intitulée "Le livre de la mémoire", ledit narrateur décide de s'appeler $A$. (p. 117) et prétend consigner sa lutte contre sa propre disparition: "Il faut qu'il s'efface afin de se trouver. Il dit donc A., même quand il pense Je" (p. 240). On pourrait 
82

supposer que ce recours à deux dispositifs énonciatifs distincts marque la distance entre le récit de la filiation biologique et le récit de la filiation textuelle. Ainsi, la reconstitution de la relation avec le père absent menée en première partie s'opposerait au parcours de la mémoire de la seconde partie, jalonné des lectures et écritures qui ont façonné l'identité du fils.

Cependant, les frontières ne sont pas si nettes et la délitescence de l'identité du fils, qui est l'objet du récit, s'accentue du fait qu'une même forme pronominale, le $i l$, sert à la fois à désigner le père - cet homme invisible dont je prétend faire le portrait - et le fils, dissimulé jusqu'à la fin derrière ce double nommé A. Le père, cet être absent, décentré, tout en surface n'est pas sans évoquer le fils, lui-même père absent d'un fils en bas âge. La permutabilité de la pronominalisation vient ici conforter l'interchangeabilité de ces identités furtives:

Le fils sauve le père. Il faut bien se représenter ceci du point de vue de l'enfant. Il faut bien se le représenter dans l'esprit du père, qui a jadis été un petit garçon, c'est-à-dire, pour son propre père, un fils. Puer ceternus. Le fils sauve le père. (p. 207)

À la fois fils et père, le je qui s'adresse à tu et devient $i l$ semble inscrire un espace énonciatif de pure forme, où les positions sont réversibles, comme au jeu de base-ball (p. 177) souvent réactivé par le texte.

La mise en évidence, par l'indifférenciation des formes pronominales, de l'identité mal assurée du personnage-écrivain s'accentue encore par le relatif anonymat des personnages de la fiction. Mon père, ma mère, ma femme, mon petit garçon, mon cousin, mon grand-père sont les figurants de cet univers familial: liés au JE par la valeur déictique du possessif, ils sont en quelque sorte les garants externes de son existence. Hors de ce réseau, et dès lors qu'il se nomme lui-même $A$., les actants sont des initiales: S., B., J., M., O., T., Q., D., P. Images des amitiés qui inscrivent le $\mathrm{A}$. dans une séquence comme autant de fragments, de reflets, de doubles de lui-même? Ou volonté expresse de refuser de nommer?

Par contraste, la dénomination des personnages de la lignée Auster s'exerce strictement à partir du père. Seul le père peut nommer et ce n'est qu'en tant que fils que les actants peuvent avoir un prénom. Ainsi, l'unique mention du nom de Paul Auster 
sera amenée dans le texte à l'occasion du coup de téléphone fictif, après la mort du père: "Paul, c'est ton père, il veut te parler" (p. 23). De la même manière, le nom du père, Sam Auster (p. 62), ne sera précisé que lors de l'évocation du procès pour le meurtre de son propre père Harry, tué par sa femme Anna (p. 65). Daniel, le fils du personnage-écrivain sera nommé dès l'ouverture du texte: “J'ai appris la mort de mon père voici trois semaines. C'était un dimanche matin, j'étais dans la cuisine en train de préparer le déjeuner de Daniel, mon petit garçon" (p. 11).

Fixée par le rapport père-fils, l'identité n'est cependant pas toujours stable. Selon la tradition judaïque, les enfants portent le nom d'un ascendant: ainsi, le même prénom désigne-t-il deux personnages. Sam le père et son oncle Samuel, Daniel le fils et Daniel Auster, le maire de Jérusalem, font écho au jeu textuel monté en parallèle autour du nom d'Anna ${ }^{2}$. Dans cette valse d'identités, même les parents du père avaient changé de nom: "Elle a rappelé qu'ils avaient vécu au Canada sóus le nom de $M$. et $M^{\text {me }}$ Harry Ball "(p. 74). Le jeu n'est pas anodin: constamment réitérée, la permutation des noms confirme que l'identité flotte à l'intérieur de l'univers familial. Je est aussi, et constamment, un autre.

À l'inverse, envisagée de l'extérieur, la question de l'identité est beaucoup plus nette. Le texte insiste sur cette dimension en nommant méticuleusement les actants secondaires. Ainsi, dans le "Portrait d'un homme invisible", où le nom du père est à peine mentionné, on désignera nommément les amitiés et les relations d'affaires du père, allant jusqu'à préciser les noms des revendeurs qui ont vidé sa maison: "quatre Noirs nommés Luther, Ulysse, Tommy Pride et Joe Sapp" (p. 22). Pareillement, l'univers de référence du fils convoque de multiples auteurs et "Le livre de la mémoire" s'écrit à l'aide de Mallarmé, Leibniz, Du Bouchet, Pascal, Tolstoï, Proust, Blanchot et d'autres ${ }^{3}$. L'individuation littéraire est

2 Les initiales d'Anna Auster, par exemple, ne sont pas sans évoquer le choix du A. qui viendra désigner le narrateur. On pourrait aussi faire valoir l'incidence du journal d'Anne Frank comme déclencheur du "Livre de la mémoire " (p. 129) lors d'une visite à Amsterdam, ou le clin d'œil de la signature de l'attestation officielle du divorce des parents, Ann W. Love (p. 111). Ce repérage non exhaustif sert à montrer comment le texte met en évidence et travaille les contours flous de l'identité.

3 L'édition française les rapatrie en un "index des citations " en fin de l'ouvrage (p. 276-280). 
fort nette et chacun des auteurs participe de la représentation d'un aspect précis du texte en train de s'écrire: un énoncé programmatique en gère à chaque fois l'apparition et le rôle. Par exemple, avec Hölderlin ${ }^{4}$, il s'agit de montrer que la certitude identitaire s'évanouit avec la solitude; après avoir précisé que Hölderlin, au terme de son mystérieux voyage à pied de Bordeaux à Stuttgart, "s'est présenté devant son ami Mathison avec ce seul mot: Hölderlin" (p. 155), le texte pointe le résultat de sa longue réclusion dans sa chambre de Tübingen:

Vers la fin, il avait l'esprit si troublé qu'il s'était mis à se donner plusieurs noms différents - Scardinelli, Killalusimeno - et un jour qu'un visiteur tardait à s'en aller, il lui a montré la porte en disant, un doigt levé en signe d'avertissement: "Je suis le Seigneur Dieu *. (p. 156)

Outre le parallélisme des situations - le narrateur-écrivain est lui aussi enfermé dans une chambre de Varick Street et s'est lui aussi donné un nouveau nom - cet extrait signale encore la distanciation du sujet par le recours à un autre auteur qui devient coénonciateur. Ce sera toutefois par la réitération du syntagme comme dans que le texte assignera aux nombreuses références convoquées le rôle de décentrement de l'instance énonciatrice ${ }^{5}$.

Une telle stratégie, dans la mesure où elle émane d'un sujet qui non seulement exprime sa quête identitaire mais recourt à d'autres textes qui mettent en scène une pareille recherche, instaure un régime d'écriture qui oscille constamment entre le témoi-

4 Je réduis sciemment l'enjeu de la référence à Hölderlin: le jeu textuel est plus complexe. Sur le plan figuratif, il apparaît comme une incarnation du parcours de A.: comme lui, il s'est enfermé dans sa chambre pour écrire, comme lui il confond sa propre identité et celles des autres, etc. La thématisation va passer par l'inscription, dans le texte, du poème de Hölderlin intitulé "A Zimmer" et faire valoir la densité de ce signifiant: "pendant trente-six ans, une bonne moitié de sa vie, il a vécu seul dans la tour que lui avait bâtie Simmer, le charpentier de Tübingen - Zimmer, ce qui, en allemand, veut dire chambren (p. 155).

5 À titre d'exemple, je retiens: "Comme dans Pascal: "Tout le malheur des hommes vient d'une seule chose, qui est de ne savoir pas demeurer au repos dans une chambre." Comme dans la phrase: "Il a écrit le Livre de la mémoire dans cette chambre." (p. 119) et encore: "C'est à ce moment-là, il s'en est rendu compte plus tard, que le Livre de la mémoire a commencé. Comme dans la phrase: "Elle a écrit son journal dans cette chambre." " (p. 129) qui permettent de bien saisir la démultiplication des structures autoreprésentatives du texte. 
gnage et la révélation du caractère construit de ce témoignage. L'invention de la solitude est-elle la transcription d'un état réel ou l'interrogation systématique des divers mécanismes de cette transcription? S'agit-il de la confrontation de deux régimes narratifs distincts, signalée par les deux parties de l'ouvrage? L'omniprésence de l'acte d'écrire, dans l'ensemble du texte, interdit d'établir une frontière nette. On peut constater, cependant, l'accentuation de la distance dans la seconde partie du texte: le travail de copiste, souventes fois mis en scène ${ }^{6}$, trouve son apogée lorsque A. affirme qu'il recopie un paragraphe de son propre texte:

\begin{abstract}
Bien qu'il ne sache pas trop ce qu'il en pense, il décide de le conserver pour référence future et le copie dans un carnet ligné:

Quand le père meurt, transcrit-il, le fils devient son propre père et son propre fils. Il observe son fils et se reconnaît sur le visage de l'enfant. Il imagine ce que voit celui-ci quand il le regarde et se sent devenir son propre père. Il en est ému, inexplicablement $[. .$.$] comme s'il marchait à la fois vers l'avant et vers$ l'arrière, dans le futur et dans le passé. Et il y a des moments, des moments fréquents, où ces sensations sont si fortes que sa vie ne lui paraît plus se dérouler dans le présent. (p. 127)
\end{abstract}

Outre la modalité évaluative marquée d'hésitation, qui rappelle la labilité de la structure identitaire, il faut lire ici le déplacement du lieu même de l'écriture. La convention établie et ponctuée dans le texte par la répétition de la phrase leitmotiv "Il pose une feuille blanche sur la table devant lui et trace ces mots avec son stylo", est déboutée; l'écriture se transporte ailleurs, dans un carnet $l i$ gné, tout en nous étant néanmoins donnée à lire. De plus, à la faveur de cette transcription qui servira de référence future, même l'axe temporel de la deixis - le maintenant de l'écriture - est déporté. Ce qu'il écrit là maintenant sera tantôt repris ici. L'identité perd ses repères: le ici de l'écriture s'exerce indifféremment là, le maintenant est reporté et le je est devenu $i l$.

Fragmenté, décentré, inventé, le sujet problématique de cette anamnèse peut-il rencontrer autre chose que des échos de sa propre parole, des reflets de lui-même? L'écriture de la mémoire

6 La co-énonciation, ainsi, transite par le relais de l'acte d'écrire : "Ensuite il ouvre un livre de Wallace Stevens (Opus Postbumous) et en copie la phrase suivante. "En présence d'une réalité extraordinaire, la conscience prend la place de l'imagination." (p. 126) 
86

n'est-elle que la construction et la déconstruction des espaces de représentation? L'exercice de solitude doit-il, à la façon d'une chambre noire, révéler le même ou l'autre? Le texte explore toutes les avenues et les laisse béantes.

\section{La chambre d'échos ${ }^{7}$}

"Tout livre est l'image d'une solitude. Un homme écrit, assis seul dans une chambre" (p. 210). Sur la foi de ces prémisses, le mécanisme analogique est enclenché et va donner lieu à un inventaire des chambres d'écriture célèbres, un peu comme si la recherche d'identité passait par la mise au jour de l'identique. L'univers rétréci aux dimensions de la chambre, lieu par excellence de la découverte de soi, est la figure de base à laquelle viendra s'indexer, au fil du texte, toute une série de modulations. Hölderlin représente entre autres, on l'a vu, les dangers potentiels de la réclusion par son expérience de la déraison alors que l'isolement cher à Pascal, à l'inverse, autorise l'exercice de la raison ${ }^{8}$. Anne Frank, de sa retraite, témoigne de la barbarie du monde (p. 130) cependant que la chambre de poète d'Emily Dickinson devient le symbole de la tradition littéraire américaine (p. 191). Ces lieux concrets du littéraire font contrepoids aux espaces représentés par Van Gogh et Vermeer où La cbambre s'oppose à La liseuse en bleu (p. 217) comme la souffrance au bonheur ${ }^{9}$. Ces reflets démultipliés de la situation inaugurale sont autant de

7 Il s'agit ici de répertorier les formes autoreprésentatives du texte. Elles sont multiples: les métaphores, mises en abyme, réduplications et figurations de la diégèse s'ajoutent aux mécanismes autoréférentiels du code signalés par l'intertextualité, les structures de surformalisation, la parodie et le recours au lexique scriptural. On aura reconnu la plate-forme proposée par Janet Paterson, dans Moments postmodernes dans le roman québécois, Ottawa, Presses de l'Université d'Ottawa, 1990.

8 À deux reprises, le texte cite Pascal: "Tout le malheur des hommes vient d'une seule chose, qui est de ne savoir pas demeurer au repos dans une chambre" (p. 119-129).

9 Cette schématisation ne rend pas justice à la complexité des liens tissês par le texte. Chacune des chambres mises en scène dans L'invention de la solitude est prétexte à une exploration ouverte des "possibilités infinies d'un espace limité " (p. 139) et l'articulation dialectique qui les réunit ici réduit leurs facteurs de correspondance. Ainsi, on pourrait faire valoir le rapport à l'écriture instauré par les tableaux: le Vermeer met en scène une liseuse, et la description de La chambre, inscrite dans le texte, est tirée de la correspondance de Van Gogh (p. 220). 
souvenirs - de visites ou de lectures - qui ponctuent cette traversée de la mémoire et sont toujours médiatisés par la figuration de l'écriture :

Il prend dans sa bibliothèque une brochure qu'il a achetée voici dix ans à Amherst, Massachusetts, souvenir de sa visite à la maison d'Emily Dickinson; il se rappelle l'étrange épuisement qui l'avait accablé ce jour-là dans la chambre du poète [...]. Il lit maintenant, à la dernière page de la brochure, dans la prose maladroite de son auteur anonyme: "Dans cette chambre-cabinet de travail, Emily proclamait que l'âme peut se satisfaire de sa propre compagnie..." (p. 190)

La distance est constamment maintenue et le système analogique recourt encore à d'autres figurations, qui forment correspondance et viennent saturer le lien entre l'écriture, la chambre et la mémoire. Les cercles concentriques de la mémoire évoquent le plan de la ville d'Amsterdam, le compteur de la voiture qui marque 67 milles correspond à l'âge du père ( 67 ans), l'enfant perdu dont fait mention le journal réactive la séparation d'avec le fils, etc. Le texte multiplie les surfaces d'inscription du réel, redouble les structures autoreprésentatives, faisant du livre de la mémoire un album d'images. Dans ce microcosme qui contient le monde, la différenciation spatiale est impertinente et la chambre de bonne de Paris est l'équivalent de la chambre de New York, dans la mesure où elle représente le lieu d'exercice de la mémoire.

Le voyage intérieur papillonne d'un objet à l'autre, un mot en fait jaillir un autre, une perception en suscite une autre, une description en évoque une autre. Les séquences combinatoires engendrent le texte, à l'image du jeu enfantin, lui-même mis en scène ${ }^{10}$. La progression événementielle est nulle, liée à la rupture de la relation de cause à effet qui gère d'habitude le narratif, les lois naturelles, sont enfreintes et seule demeure la pensée

10 "A. a parfois l'impression que les démarches mentales de son fils en train de jouer sont l'image exacte de sa propre progression dans le labyrinthe de son livre. Il a même imaginé que s'il arrivait à représenter par un diagramme les jeux de son fils (une description exhaustive, mentionnant chaque déplacement, chaque association, chaque geste) et son livre par un autre, similaire (en élucidant ce qui se passe entre les mots, dans les interstices de la syntaxe, dans les blancs entre les paragraphes - en d'autres termes en démêlant l'écheveau des connexions), les deux diagrammes seraient identiques: ils se superposeraient parfaitement " (p. 258) 
associative. Statique, le texte apparie, rapproche, compare, mettant en images les rouages de la pensée en train de s'écrire ${ }^{11}$.

"La mémoire: une chambre, un crâne, un crâne qui renferme la chambre dans laquelle un corps est assis" (p. 138). La mise en abyme inscrit ici un axe de correspondances verticales et l'engendrement horizontal de scènes similaires - chaque chambre en évoquant une autre - se double d'un emboîtement de récits parallèles, reproduisant la structure des Mille et une nuits. Non seulement faut-il écrire pour ne pas mourir, mais chacune des histoires racontées doit illustrer le schéma narratif premier. Ici, la pensée du même stratifie le matériau textuel en superposant les images de l'enfermement dans "le ventre de la baleine". L'intertextualité, subordonnée à l'enjeu d'illustration, joue d'abondance de deux textes majeurs, Le livre de Jonas et Les Aventures de Pinoccbio de Collodi, "qui s'appelait en vérité Carlo Lorenzini" (p. 256).

Après avoir fait saillir le lien analogique entre Le livre de Jonas et "Le livre de la mémoire" sur le plan formel ${ }^{12}$, le texte va tisser un réseau de similitudes qui vont condenser la judaïté et le rapport père-fils. Tressée autour de l'image de l'enfermement, l'identité des deux textes joue sur plusieurs registres qui tous reflètent la difficile accession à la parole. Ainsi l'expérience de la solitude délie la langue de Jonas, qui avait initialement refusé l'injonction de Iahvé (193-195), tout comme la réclusion de A. autorise l'écriture; dans les deux cas, la fuite les mène aux limites du monde connu et le silence implique à la fois un refus de l'autre et une interrogation à propos de la vérité de la parole.

Outre ces parentés diégétiques qui lient Jonas et le narrateur A., c'est cependant davantage le statut du texte sacré qui sanc-

11 Les quatre étapes majeures de la cognition, au sens de Georges Vignaux, sont l'identité, la séparation, l'association et l'inclusion (Le discours acteur du monde. Énonciation, argumentation et cognition, Paris, Ophrys, 1988). On l'aura compris, je joue ici librement de ces quatre variables, les départageant au gré des divers plans de l'analyse.

12 "Le livre de la mémoire. Livre sept. Premier commentaire sur le Livre de Jonas. On est frappé dès le premier abord par sa singularité dans l'ensemble des textes prophétiques. Cette œuvre brève, la seule qui soit écrite à la troisième personne, est la plus dramatique histoire de solitude de toute la Bible, et pourtant elle est racontée comme de l'extérieur, comme si plongé dans les ténèbres de cette solitude, le "moi" s'était perdu de vue. Il ne peut donc parler de lui-même que comme d'un autre. (193) 
tionne le parallèle. Lié à la plus grande solennité du calendrier juif - la fête du Yom Kippour c'est-à-dire du Grand Pardon- le minuscule Livre de Jonas, "étrange et parfois comique" donne lieu à des exégèses contradictoires, inscrites dans le texte (p. 247249). Ces lectures érudites de Théodore de Mopsueste, du rabbin Akiba et de Rupert de Deutz accentuent la dimension politique de l'aventure de Jonas, le seul des prophètes qui doit s'adresser à des non-juifs. Dans cette perspective, Le livre de Jonas cristallise la question de l'identité juive menée sur d'autres axes du texte par la réactivation constante de l'holocauste et vient répondre à l'assertion de la poète Marina Tsvetaieva selon laquelle: "En ce monde-ci hyperchrétien/Les poètes sont des juifs" (149).

Une autre histoire de solitude exploitée par le texte est celle de Pinocchio. Au gré de l'histoire racontée à son fils Daniel, le texte met en lumière la dimension psychologique de la quête entreprise par le narrateur. Gepetto et Pinocchio sont séparés, tout comme A. est à la fois séparé de son père et de son fils, et Pinocchio ne pourra devenir un vrai garçon que lorsqu'il aura retrouvé son père. L'évidence grossière de la ressemblance, amorcée par l'histoire de Disney, va se raffiner lors de la lecture de la version de Collodi. Là où l'adaptation de Disney sentimentalise et banalise les motivations profondes du désir d'être en les exprimant, l'original de Collodi laisse advenir la conscience de soi, sans recommandation ni directive. Mais ce sera surtout, on le devine, par la mise en scène du travail de l'écriture que la supériorité du texte de Collodi se signalera:

En plongeant sa marionnette dans l'obscurité du requin, Collodi nous le dit, il plonge sa plume dans le noir de son encrier. Pinocchio, après tout, n'est fait que de bois. Collodi l'utilise comme un instrument (littéralement: un porte-plume) pour écrire sa propre histoire. Ceci sans nulle complaisance pour une psychologie primaire. Collodi n'aurait pu réussir ce qu'il a entrepris avec Pinocchio si le livre n'avait été pour lui un livre de mémoire. (p. 255)

L'autoreprésentation est nette et "Le livre de la mémoire" s'écrit en une succession de miroirs littéraires qui sont autant de facettes de l'éveil à soi par l'écriture.

L'espace de la chambre s'incarne encore dans des réduplications diégétiques, cette fois fortement dialectisées. La relation insatisfaisante de $A$. avec son père trouve compensation dans le 
rapport qui s'établit entre A. et S. ${ }^{13}$. À titre de figure substitutive du père, le personnage $S$. en est l'image strictement inversée et le contraste va être montré dans le texte par la représentation de leur espace respectif. D'une part, la maison de style Tudor et ses pièces aux proportions royales, en état de négligence poussé, qui la fait apparaître, avec ses fenêtres aux stores toujours baissés, comme l'antre d'un aveugle: "elle était devenue métaphore de la vie de mon père, représentation exacte et fidèle de son monde intérieur" (p. 17). D’autre part, le réduit obscur de S., à la fenêtre tendue d'une épaisse étoffe noire, qui lui permet de rêver les yeux ouverts: "c'était une châsse, à peine plus grande qu'un corps humain, à la gloire de tout ce qui en dépasse les limites: la représentation, jusqu'au moindre détail, du monde intérieur d'un homme" (p. 140). Lés mêmes termes servent à marquer la différence.

La figure idéale du père, représentée par S., est celle d'un être excentrique, en marge de la méchanceté du monde, tout entier consacré à la réalisation de son ouvre, une "composition pour trois orchestres et quatre chœurs dont l'exécution aurait duré douze jours" (p. 141). Le projet, qualifié de Work in Progress en un écho conscient à Joyce, est aussi décrit comme "l'ouvrage-à-accomplir-qu'on-accomplit-en-l'accomplissant" (p. 143), sans qu'intervienne la nécessité de le mener à terme. Au contraire, la valeur de vérité de l'entreprise tient justement à l'impossibilité de sa réalisation. Le parallèle avec l'œuvre de A. réside dans la conscience de l'absurdité de sa propre démarche, qui entend reconstituer le portrait d'un homme invisible à la faveur de quelques traces insignifiantes et éparses, remises en ordre par la voix de la mémoire, "parole de Cassandre dont nulle leçon n'est à tirer, parole, somme toute, pour ne rien dire, et dont nous ne faisons que prendre acte, sans conclure, dès lors que nous l'avons entendue" (p. 199). Du fond de sa cellule solitaire, Cassandre monologue, alignant dixsept cents vers, prise par le tournoiement de sa propre parole:

Dans ce poème dense, déconcertant, rien n'est jamais nommé, tout devient référence à autre chose. On se perd rapidement

13 Le texte, une fois de plus, est très explicite: "S. et A. étaient attirés l'un vers l'autre par des besoins convergents: pour l'un, celui d'un fils qui l'acceptât tel qu'il était; pour l'autre, celui d'un père qui l'acceptât tel qu'il était. Ceci était renforcé par des coïncidences de dates: $S$. était né la même année que le père de A., qui était né la même année que le plus jeune fils de S. " (p. 144) 
dans le labyrinthe de ces associations, et pourtant on continue à le parcourir, mû par la force de la voix de Cassandre. (p. 199)

Le recours au Cassandre de Lycophron sert encore à étayer, si besoin était, l'identité de traducteur de A. et à tisser plus étroitement encore la filiation textuelle. D'une voix à l'autre, de Lycophron à Royston à Q. ${ }^{14}$, la parole est transmise à travers les âges et les langages, du grec à l'anglais, au français et à nouveau à l'anglais, au fil des traductions et des reprises, et le lieu de la solitude devient une vertigineuse chambre d'échos.

La musique du hasard ${ }^{15}$ n'assourdit pas ses harmoniques et le texte va s'employer à faire rimer les mots, les événements, les dates, les lieux, les naissances, les morts, les textes. La mémoire est un "espace dans lequel un événement se produit pour la seconde fois" (p. 130) et l'écriture austérienne joue à l'infini des variations du même. Les situations appariées sont multiples, signalant du coup la déconstruction de la représentation:

Le 23 janvier 1919, soixante ans exactement avant la mort de mon père, sa mère a tué son père d'un coup de feu dans la cuisine de leur maison, avenue Frémont à Kenosha, Wiconsin. (p. 58)

Cette coïncidence majeure est répercutée sur le mode mineur par la succession des hasards qui viennent rythmer le réel. Le fils de A. est né le même jour qu'Anne Frank (p. 130), l'ami américain $M$. loue à Paris une chambre de bonne qui s'avère être celle où s'était réfugié son propre père pendant la guerre quelque vingt ans plus tôt (p. 125), le rabbin qui préside aux funérailles du père est le même qui avait officié lors de la Bar Mitzvab de A. (p. 109), la même note, le fa du milieu, manque à deux pianos différents (p. 225). Même l'anodin est récupéré et l'année 1893 est à la fois l'année de naissance du grand-père et celle de la modification de la géométrie du terrain de base-ball (p. 179): "Coïnci-

14 Révélation: le Q., ce personnage énigmatique à l'origine de la découverte du poème de Lycophron, est Pascal Quignard, qui l'a traduit, sous le titre Alexandra du grec au français. C'est toujours Q. qui lui fera connaître la traduction anglaise de Lord Royston, au siècle dernier. (197)

15 Je reprends ici le titre d'un autre ouvrage de Paul Auster, paru en français chez Actes Sud en 1991. Cet ouvrage a donné lieu à un film, portant le même titre, et mis en scène par Philip Haas, dans lequel Paul Auster tient un petit rôle, sans avoir collaboré à l'écriture du scénario. Pour le détail des activités cinématographiques de Paul Auster, on peut lire l'entrevue de Florianne Vidal, "Paul Auster léviathan", parue dans Art Press, n 179 , en avril 1993. 
92

dence: ce qui survient avec. Ce qui occupe le même point dans le temps ou l'espace" (p. 254).

L'invention de la solitude, on l'a vu, est un recueil d'histoires de vie et de mort. Un jeu de différenciations inscrit des nuances entre la mort sans préavis du père, seul dans son fauteuil, et celle du grand-père maternel, après deux semaines d'hospitalisation où A. lui rend visite tous les jours. La naissance du fils de la poète russe, en l'absence de son père, est la réplique de la propre naissance de A., et c'est ce même fils qui figure sur la photo au mur de $\mathrm{S}$. Le regard qui rapproche constamment les éléments du passé personnel tisse en même temps une trame où l'histoire du monde se répète : "Le Dernier Testament d'Israël Licbtenstein" répond à la lettre de Nadejda Mandelstam et les enfants du Cambodge sont les échos des enfants de Bergen-Belsen.

Il ne s'agit pas ici de faire l'inventaire des réduplications diégétiques du texte austérien: elles sont infinies, et se voient encore démultipliées par la fragmentation narrative qui fait que les mêmes événements sont racontés deux fois ${ }^{16}$. Un tel effet d'insistance marque le caractère tensif qui existe entre la quête de sens et la distanciation ironique, qui font toutes deux l'objet d'une mise en scène. De fait, le démiurge qui préside à toutes ces connexions n'est jamais dissimulé, et ses stratégies combinatoires, qui confinent parfois au grotesque, sont autant d'affirmations de l'esthétisation de son dire:

De même que les mots, les choses ne prennent un sens que les unes par rapport aux autres. "Deux visages semblables, écrit Pascal, dont aucun ne fait rire en particulier, font rire ensemble par leur ressemblance". Ces visages riment pour l'œil, juste comme deux mots peuvent rimer pour l'oreille. Poussant un peu plus loin, A. irait jusqu'à soutenir que les événements d'une vie peuvent rimer entre eux [...] et la rime qu'ils produisent quand on les voit ensemble modifie la réalité de chacun d'eux. (p. 252)

L'intention avouée de modifier la réalité par le rapprochement du semblable trouve encore à s'exercer par l'évocation textuelle. À

16 À cet égard, la parution récente du Carnet rouge (Arles, Actes Sud, 1993), lequel, incidemment, n'est pas sans évoquer "le souple carton rouge abritant les notes de Mallarmé ", est extrêmement révélatrice : non seulement cet ouvrage est-il le florilège des cö̈ncidences de la "vie" de Paul Auster, mais il renvoie aux coïncidences mises en scène dans ses ouvrages précédents. 
ce titre, on pourrait lire dans les fragments Pour un tombeau d'Anatole de Mallarmé - qui figurent en fin d'ouvrage - une base génératrice de L'invention de la solitude, qui deviendrait ainsi le Tombeau du père disparu ${ }^{17}$. L'allusion, par trop évidente, se voit encore bridée de multiples fils narratifs : la maladie du fils de A., la rencontre d'une lettrée américaine qui édite Mallarmé, sa propre traduction des fragments, "en décembre 1979, cent ans exactement après que Mallarmé eut composé ces pages funèbres à son fils"(p. 172), traduction parue dans Paris Review avec la photo d'Anatole Mallarmé et que l'ami R., feuillettant la revue par hasard, confondra avec le propre fils de A., etc. Ces raccords vont permettre le saut du narratif au discursif en devenant le prétexte d'un "commentaire sur le mot rayonnement" (p. 174). La mise en mots de la mort du père, littéralement, devient le poème de son existence.

Fondus au creuset de l'analogie, les événements qui riment comme des mots sont le décalque de l'exercice littéraire. Le sujet est un être de papier, d'écriture, un être de parole sensible à la grammaire de son existence qui "comporte tous les aspects du langage: comparaison, métaphore, métonymie, synecdoque" (p. 253). D'entrée de jeu, le passage de la vie à la mort apparaît comme une modification de la syntaxe : l'individu vivant est synonyme de son corps alors que la mort va les dissocier (p. 24). Entre la sémantique et la syntaxe, le texte jouera encore d'allitérations et d'assonances, s'amusera de rapprochements de signifiants (le jeune Georges Efron était au front), réfléchira sur un jeu de rimes anglaises (womb et tomb, breatb et deatb) jusqu'à saturer tous les plans du langage. Cet enchaînement d'échos sonores se retrouve également dans la structuration des paragraphes, souvent encadrés de phrases similaires, comme s'il s'agissait d'épuiser toutes les combinaisons possibles. Plus spectaculaire encore sera l'anaphorisation du syntagme Il se souvient, qui inaugure

17 Dans "Le fils de Mallarmé ", Paul Auster explique en ces termes la motivation de Mallarmé: "Par la transmutation qu'il ferait d'Anatole en mots, il prolongerait sa vie. Il le ressusciterait, littéralement, puisque le fait d'édifier un tombeau - un tombeau poétique - effacerait la présence de la mort [...] et ce ne serait que lorsque Mallarmé luì-même disparaîtrait que l'enfant disparấtrait avec lui. Ces notes constituent l'une des relations les plus émouvantes des efforts d'un homme pour affronter la mort moderne - c'est-à-dire la mort sans Dieu, la mort sans espoir de salut", L'art de la faim, Arles, Actes Sud, 1992 [trad. française], p. 209. 
94

chacune des phrases des cinq premières pages du Livre treize. Le son, la lettre, le lexique, la grammaire, la sémantique, l'encyclopédie sont mis à contribution dans cette valse de l'identique où la vie est le langage et forme système de proche en proche avec l'univers entier. Tel est le monde plein de l'écriture austérienne, où tout est le miroir de tout et qui, de réduplications en répétitions, de rimes en métaphores, invente une solitude qui n'en est pas une. S'il est un art poétique qui s'affiche, et en cela réside le paradoxe, ce sera celui de la monadologie leibnizienne:

Et par conséquent tout corps se ressent de tout ce qui se fait dans l'univers, tellement que celui qui voit tout pourrait lire dans chacun ce qui se fait partout, et même ce qui s'est fait ou se fera, en remarquant dans le présent ce qui est éloigné tant selon les temps que selon les lieux. Mais une âme ne peut lire en elle-même que ce qui y est représenté distinctement; elle ne saurait développer tout d'un coup ses replis, car ils vont à l'infini. (Leibniz, p. 251)

Dans la solitude de cette chambre noire qui est le lieu du devenir, le sujet habité de langage n'est jamais seul.

\section{Uhneimlich ou heimlich?}

Amorcé par le récit de la mort réelle du père, L'invention de la solitude s'achève sur le récit de la mort rêvée du fils. Cette homologie va sanctionner la séparation ultime, et les échos pourront enfin se taire. Du portrait au livre, la voix aura, à l'injonction de Kierkegaard, enfanté le père. Les deux parties du texte s'aboutent et là où il s'agissait de décrire pour en finir, le geste d'écrire permettra de se souvenir. Certes, sur le plan narratif, l'enjeu consiste à combler un réel vide par l'exercice de la parole; en même temps, cependant, le passage du voir au dire correspond à la textualisation de la différence entre la vie et l'œuvre d'art.

Cette différence sera thématisée entre autres par le recours aux représentations photographiques et picturales. Une double médiatisation s'instaure: le narrateur regarde et commente les clichés de sa famille et quelques portraits célèbres, inscrivant la subordination du réel à sa représentation. L'opposition entre les centaines de photos éparses, négligemment jetêes dans des enveloppes fanées au fond des tiroirs et les tableaux de collection, pieusement conservés dans des musées, marque grossièrement la frontière entre le témoignage de la vie et la démarche artistique. 
Se jouant de ce clivage convenu entre la photo et le tableau, le texte va s'ingénier à en intervertir les codes de lecture.

Ainsi, l'album de famille titré à l'or fin "Ceci est notre vie: les Auster" (p. 23) accuse, du fait de sa vacuité, la viduité de la vie du père. Mais il y a plus: chacun des clichés commentés devient facteur d'irréalité. La photo de la mère, en voyage de noces aux chutes du Niagara, juchée nerveusement sur un taureau pour un de ces clichés amusants qui n'amusent personne (p. 19), répond aux photos du père, prises avant son mariage, où les poses comiques sur fond de court de tennis ou de piscine accentuent le caractère superficiel du personnage (p. 26). Deux autres photos, reproduites dans une édition américaine de l'ouvrage ${ }^{18}$, insistent encore sur la facticité de ces images du réel. La première est une photo truquée qui représente le père démultiplié en cinq exemplaires assis autour d'une table:

Il est là cinq fois, mais la nature du trucage rend impossible tout échange de regards entre les personnages. Chacun est condamné à fixer le vide, comme sous les yeux des autres, mais sans rien voir, à jamais incapable de rien voir. C'est une représentation de la mort, le portrait d'un bomme invisible. (p. 52)

La seconde photo, trafiquée celle-là, conserve néanmoins la trace d'un autre homme invisible: sciemment déchirée et recollée de façon à faire disparaître l'image du grand-père assassiné par sa femme, cette photo ne garde que le bout de ses doigts, "comme s'il avait été exilé dans une autre dimension" (p. 55). La perversion est nette: là où la photo doit dire le réel, elle ne fait que le masquer. Le familier, le beimlich de Freud (p. 230) devient ainsi unbeimlich, porteur d'une inquiétante étrangeté.

À l'inverse, l'œuvre d'art va témoigner du réel. Les portraits successifs peints par Rembrandt de son fils Titus, mort avant lui, disent le regard pénétrant du peintre (p. 153). De la même manière, le tableau (d'auteur inconnu) représentant Sir Walter Raleigh avec son fils Wat à huit ans montre la similitude de leurs attitudes: "l'expression de farouche détermination du fils imite le regard assuré et impérieux du père"(p. 153). L'acte de transposi-

18 The Invention of Solitude, reparu en $1988 \mathrm{chez}$ Penguin Book reproduit en page couverture la photo truquée du père et reprend, avant le début du texte, en page 4 , l'intrigante photo falsifiée d'où le grand-père a été carrément éliminé. 
tion, ici, n'éconduit nullement la réalité, il la rend perceptible. À preuve, le traitement textuel du tableau La chambre de Van Gogh, qui lui confère le statut d'autoportrait: "il s'agit bien d'un autoportrait, semblable à n'importe quelle représentation du visage d'un homme avec nez, yeux, lèvres et mâchoire" (p. 221).

Un tel procédé, qui abolit la frontière entre la photo et le tableau, est intimement lié, on le pressent, à la représentation de l'acte de lecture ${ }^{19}$. L'effet esthétique réside dans la différenciation du regard. Lire le réel en colligeant les coïncidences, tout comme écrire le réel en multipliant les réduplications, sont les volets d'une même démarche, qui interpelle le statut du texte:

Dans une ouvre de fiction, on admet l'existence, derrière les mots sur la page, d'une intelligence consciente. Rien de pareil en présence des événements du monde prétendu réel. Dans une histoire inventée, tout est chargé de signification, tandis que l'histoire des faits n'a que celle des faits eux-mêmes. (p. 226)

L'invention de la solitude est un acte de construction, une mise en forme du réel qui cherche la signification de la mort du père. À défaut de pouvoir la lire, il faudra l'inventer, la construire. La spécification des registres discursifs entre le "Portrait d'un homme invisible" et "Le livre de la mémoire" entendait dresser le regard du lecteur, lui apprendre à non plus départager, mais bien à confondre l'espace de la fiction et l'espace du réel. De fait, la représentation de l'acte d'écrire, constamment réitéré par la segmentation du livre en programmes d'écriture, risquait de miner l'authenticité du portrait diégétique. Le jeu de la mémoire, en s'affichant comme une "structure artificielle d'ordonnancement pour le passé historique" (p. 178), inverse la polarité et la narrativisation de l'invisible s'actualise par la représentation du lisible. La diégèse se tisse au gré des méandres discursifs, problématisant et affichant, jusqu'à la toute fin, l'indétermination du sens :

19 Max Roy, dans "Stratégies de lecture dans le roman contemporain", décrit en ces termes l'effet d'une telle représentation: "À travers des pratiques inédites ou hybrides qui peuvent être qualifiées de postmodernes, notamment au sens où elles utilisent à leurs propres fins des procédés modernes, la prise en charge du procès de lecture dans le texte de fiction constitue une perspective esthétique et critique séduisante parce que ses effets sont immédiatement interprétables" (Tangence, $n^{\circ} 39$, "La fiction postmoderne ", p. 87). En l'occurrence, la représentation de l'acte de lecture apparât comme une ficelle supplémentaire, qui désigne la faillite de l'appropriation du réel. 
Le ciel est bleu, noir, gris, jaune. Le ciel n'est pas là, et il est rouge. Tout ceci s'est passé hier. Tout ceci s'est passé voici cent ans. Le ciel est blanc. Il a un parfum de terre, et il n'est pas là. Il est blanc comme la terre, et il a l'odeur d'hier. Tout ceci s'est passé demain. Tout ceci s'est passé dans cent ans. Le ciel est citron, rose, lavande. Le ciel est la terre. Le ciel est blanc, et il n'est pas là. (p. 270)

L'invention de la solitude, régie par une esthétique de la correspondance qui cumule le semblable, mène à la déréalisation. De l'intuition du réel à sa construction, le parcours cognitif du texte s'achève sur l'indétermination: le $\mathrm{A}$. ne se réunifie pas dans le je, la trame narrative est contestée et l'écriture réaffirme son strict statut de langage.

L'espace subjectif, schématisé pour fins d'analyse en espaces identitaire, référentiel, dialogique, mémoriel et textuel, pulvérise le noyau énonciatif et fragmente le dire à l'infini. Loin de se fondre en un lieu unique, faisant du cogito le maître de la pensée et du langage, les espaces se chevauchent, ouvrant des interstices où le sens s'engouffre.

Le marionnettiste solitaire qui préside à cette mise à distance de soi déjoue la fiction autobiographique par le jeu inlassablement repris des aléas du hasard et par l'invention de séquences associatives de plus en plus complexes. Le magicien, en l'occurrence, n'est pas dupe et s'évertue à montrer que "le langage n'est pas la vérité. Il est notre manière d'exister dans l'univers" (p. 251). 